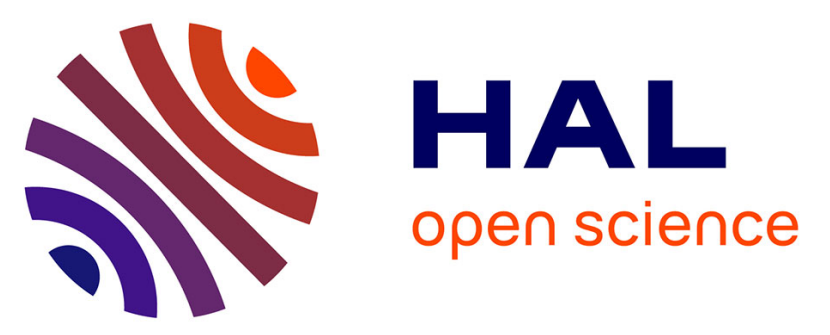

\title{
On-demand pre-exposure prophylaxis with tenofovir disoproxil fumarate plus emtricitabine among men who have sex with men with less frequent sexual intercourse: a post-hoc analysis of the ANRS IPERGAY trial
}

Guillemette Antoni, Cécile Tremblay, Constance Delaugerre, Isabelle Charreau, Eric Cua, Daniela Rojas Castro, François Raffi, Julie Chas, Thomas Huleux, Bruno Spire, et al.

\section{- To cite this version:}

Guillemette Antoni, Cécile Tremblay, Constance Delaugerre, Isabelle Charreau, Eric Cua, et al.. Ondemand pre-exposure prophylaxis with tenofovir disoproxil fumarate plus emtricitabine among men who have sex with men with less frequent sexual intercourse: a post-hoc analysis of the ANRS IPERGAY trial. The Lancet HIV, 2020, 7 (2), pp.e113-e120. 10.1016/S2352-3018(19)30341-8 . inserm03203315

\section{HAL Id: inserm-03203315 https://www.hal.inserm.fr/inserm-03203315}

Submitted on 20 Apr 2021

HAL is a multi-disciplinary open access archive for the deposit and dissemination of scientific research documents, whether they are published or not. The documents may come from teaching and research institutions in France or abroad, or from public or private research centers.
L'archive ouverte pluridisciplinaire HAL, est destinée au dépôt et à la diffusion de documents scientifiques de niveau recherche, publiés ou non, émanant des établissements d'enseignement et de recherche français ou étrangers, des laboratoires publics ou privés. 
On-demand PrEP with TDF/FTC remains effective among men who have sex with men with infrequent sexual intercourses. A sub-study of the ANRS IPERGAY trial.

\section{Authors}

Guillemette Antoni(1), Cécile Tremblay(2), Constance Delaugerre(3,4,5), Isabelle Charreau(1), Eric Cua(6), Daniela Rojas Castro(7,8,9), François Raffi(10,11), Julie Chas(12), Thomas Huleux(13), Bruno Spire(8,9), Catherine Capitant(1), Laurent Cotte(14), Laurence Meyer $(1,15)^{\mathrm{f*}}$ and Jean-Michel Molina $(3,4,5)^{*}$ and the ANRS IPERGAY study group**.

${ }^{\mathrm{f}}$ corresponding author

*contributed equally

(1) INSERM SC10 US19, Villejuif, France

(2) Centre Hospitalier de l’Université de Montréal, Canada

(3) Hôpital Saint-Louis, Assistance Publique Hôpitaux de Paris, France

(4) INSERM UMR 944, Biologie cellulaire des infections virales, Paris, France

(5) Université de Paris, France

(6) Hôpital de l'Archet, Nice, France

(7) Coalition PLUS, Pantin, France

(8) Aix Marseille University, INSERM, IRD, SESSTIM, Sciences Economiques \& Sociales de la Santé \& Traitement de l'Information Médicale, Marseille, France

(9) ORS PACA, Observatoire régional de la santé Provence-Alpes-Côte d’Azur, Marseille, France

(10) INSERM CIC 1413

(11) Service des Maladies Infectieuses et Tropicales, Centre hospitalier Universitaire de Nantes, France

(12) Hôpital Tenon, Paris, France

(13) Hôpital G. Dron, Centre Hospitalier Universitaire de Tourcoing, France

(14) Hôpital de la Croix Rousse, Hospices Civils de Lyon, France

(15) Université Paris Sud

** Other investigators in the ANRS IPERGAY study group are listed in the Acknowledgments. 


\section{Keywords}

On-demand PrEP, Efficacy, MSM, Infrequent sexual intercourse

\section{Research in context}

\section{Evidence before study}

Pre-exposure prophylaxis (PrEP) of HIV infection with oral tenofovir/emtricitabine (TDF/FTC) is highly effective among high-risk men who have sex with men (MSM) with daily (Iprex [1], Proud [2]) or ondemand regimen (Ipergay [3]). The open-label extension of the Iprex trial reported no incident HIVinfection in individuals taking at least 4 pills per week [7]. The on-demand dosing regimen in the ANRS Ipergay trial requested the intake of 4 pills to cover one sexual intercourse. Participants reported a median of 10 sexual acts per month (i.e. 2.5 /week) and a median intake of 15 pills per month (i.e. nearly 4 pills/week) [3]. Therefore, it was unclear whether the efficacy reported during the ANRS Ipergay trial was not merely due to the repeated use of 4 pills per week with enough accumulation of active drugs. However, monkeys and pharmacokinetics studies argue in favour of efficacy of eventdriven PrEP $[14,16]$. We searched on PubMed until April 31, 2019 with the terms ((PrEP) OR (Preexposure prophylaxis)) AND ((on-demand) OR (event driven)). We identified 65 studies: none of them evaluated the efficacy of on-demand PrEP in case of infrequent sexual intercourse. We therefore investigated whether on-demand PrEP remained effective among MSM having infrequent sexual intercourses with high PrEP adherence during the randomized double-blind placebo-controlled ANRSIPERGAY trial.

\section{Added value of this study}

Based on data collected in the double-blind phase of the IPERGAY trial, we identified periods of followup between visits during which participants took 15 pills or less per month but used them systematically or often during sexual intercourses, as a proxy of infrequent sexual intercourses covered by PrEP. During these 134 person-years of follow-up, the participants used a median of 9.5 pills per month and had a median of 5 sexual intercourses per month, i.e. around 2.2 pills and less than 1.2 intercourses per week, on average. Six infections occurred in the placebo arm (HIV incidence: 9.2 infections per 100 person-years; 95\% Cl: 3.4-20.1) versus 0 in the TDF/FTC arm (HIV incidence: 0; 95\% $\mathrm{Cl}: 0.0-5.4), \mathrm{p}=0.013$, leading to a relative reduction of HIV incidence of $100 \%$ (95\% Cl: $39-100)$.

\section{Implications of all the available evidence}

This study provides evidence that sex-driven PrEP is an adequate alternative to daily PrEP for high-risk MSM even during periods of infrequent sexual intercourses. The choice of the regimen (daily or sexdriven) should be offered to all MSM subjects, who could therefore adapt their uptake according to 
59 their evolving sexual lives and their lives context. The efficacy of on-demand PrEP in persons with very 60 low sexual activity such as once per month could not be stated here given the size of the sample and 61 should be confirmed in current open studies of on-demand PrEP. 


\section{Abstract}

63

64

65

66

67

68

69

70

71

72

73

Background: The randomized double-blind ANRS-IPERGAY trial demonstrated among high-risk men who have sex with men (MSM) a major reduction of HIV-1 incidence with on-demand PrEP with TDF/FTC as compared to placebo. During the trial participants used a median of 15 pills of TDF/FTC per month and had a median of 10 sexual intercourses per month. We wished to investigate whether ondemand PrEP remained effective among MSM having less frequent sexual intercourses and using therefore fewer pills.

Methods: We focused our analysis on periods during which participants took 15 pills or less per month but used them "systematically or often during sexual intercourses" as a proxy of infrequent sexual intercourses covered by PrEP. We then cumulated in each arm follow-up time spent with this pattern of PrEP use. To estimate a window time of HIV acquisition during the study, results of $4^{\text {th }}$ generation HIV-1/2 ELISA assays and plasma HIV-1 RNA levels from frozen samples, Western Blot and Fiebig staging were reviewed blindly. Incidence rates of HIV-infection per 100 person-years (py) were compared between the two arms by using mid-p exact test.

Findings: Two hundred and seventy participants have used 15 pills/month or less between two visits at least once during the study, with PrEP being used systematically or often during sexual intercourses, representing 134 py of follow-up and $31 \%$ of the total study follow-up. During these periods, participants reported a median of 5 (IQR: 2-10) sexual intercourses/month and used a median of 9.5 (IQR: 6-13) pills/month. Six HIV-1 infections were diagnosed: 6 in the placebo arm (HIV incidence: 9.2 infections per 100 py; 95\% Cl: 3.4-20.1) and 0 in the TDF/FTC arm (HIV incidence: 0; 95\% Cl: 0.0-5.4), $p=0.013$, with a relative reduction of HIV incidence of $100 \%$ (95\% Cl: $39-100)$.

Interpretation: On-demand PrEP with TDF/FTC remained highly effective in MSM having infrequent sexual intercourses. 


\section{Introduction}

Pre-exposure prophylaxis (PrEP) of HIV infection with oral tenofovir/emtricitabine (TDF/FTC) is highly effective among high-risk men who have sex with men (MSM). Relative reduction in incidence rates conferred by daily PrEP was $44 \%$ in the iPrex trial ${ }^{1}$ and reached $86 \%$ in the Proud study ${ }^{2}$. The ANRS Ipergay trial which evaluated the efficacy of on-demand PrEP with TDF/FTC, i.e. driven by sex events (two pills 2 to 24 hours before sex, followed by a third pill 24 hours after the first drug intake and a fourth pill 24 hours later), found an $86 \%$ relative reduction of HIV incidence compared to placebo ${ }^{3}$. Based on these data, both daily and on-demand PrEP regimens are proposed to MSM in Europe but $^{4}$ the on-demand dosing regimen is not yet endorsed by CDC or WHO because of uncertain efficacy among participants having infrequent sexual intercourses ${ }^{5,6}$. Indeed, since the on-demand dosing regimen in the ANRS Ipergay trial requested the intake of 4 pills to cover one sexual intercourse, and since the open-label extension of the Iprex trial ${ }^{7}$ reported no incident HIV-infection in individuals taking at least 4 pills per week, it was unclear whether the efficacy reported during the ANRS Ipergay trial was not merely due to the repeated use of 4 pills per week with enough accumulation of active drugs. Participants reported indeed a median of 10 sexual acts per month (i.e. 2.5 /week) and a median intake of 15 pills per month (i.e. nearly 4 pills/week) during the ANRS Ipergay trial.

We therefore wished to investigate whether on-demand PrEP remained effective among MSM having less frequent sexual intercourses with high PrEP adherence and using therefore fewer pills during the ANRS Ipergay trial.

\section{Methods}

\section{The ANRS IPERGAY trial}

The ANRS IPERGAY trial has already been reported ${ }^{3}$. Briefly, this double-blind, randomized trial of ondemand PrEP enrolled HIV-negative adult MSM or transgender women who had condomless anal sex with at least two partners during the past six months ${ }^{3}$. Participants were assigned in a 1:1 ratio to 
receive either TDF/FTC or placebo. TDF/FTC was given as a fixed-dose combination of $300 \mathrm{mg}$ of TDF and $200 \mathrm{mg}$ of FTC per pill. Participants were instructed to take a loading dose of two pills of TDF/FTC or placebo 2 to 24 hours before sex, unless the last drug intake was less than 1 week earlier in which case they were instructed to take only one pill, followed by a third pill 24 hours after the first drug intake and a fourth pill 24 hours later. In case of multiple consecutive sexual intercourses, participants were instructed to take one pill per day until the last sexual intercourses and then to take the two post exposure pills.

The protocol was approved by public health authorities and ethics committees in France (Comité de Protection des Personnes lle de France IV) and Canada (Comité d'Ethique de la Recherche de Montreal). All participants provided written informed consent.

Participants had a screening visit followed by the inclusion visit (DO) one month later; next visits were scheduled at month one (M1), two (M2), and every two months thereafter. The protocol required serum and plasma storage at $-80^{\circ} \mathrm{C}$ at each study visit. Drugs were dispensed at each visit with enough pills to cover the daily use of TDF/FTC or placebo between visits. Participants were asked to return their bottles at each visit. A pill count of unused medication was performed, allowing assessment of the number of pills used per month. Pills uptake was also estimated by tenofovir concentration from frozen plasma at each visit, with a limit of quantification of $1 \mu \mathrm{g} / \mathrm{L}^{8}$. At each visit during a face-to-face interview with the physician, participants were asked if they had used PrEP since last visit: "systematically, respecting the dosing recommendation", "for each sexual period, but not fully respecting dosing recommendations", "often, respecting dosing recommendations", "often, not fully respecting dosing recommendations", "from time to time, respecting dosing recommendations", "from time to time, not fully respecting dosing recommendations", or "not at all". At each visit, the number of sexual acts during the previous month as well as the number of sexual partners since the previous visit were recorded in a computer-assisted structured interview completed online by the participants. 
The primary end point was the diagnosis of HIV-1 infection. During the trial, a fourth-generation enzyme linked immunosorbent assay (ELISA-4G) for HIV-1 and HIV-2 combined was performed at each scheduled visit and anytime in case of suspicion of primary HIV infection, using the ARCHITECT HIV $\mathrm{Ag} / \mathrm{Ab} \mathrm{Combo}^{\circledR}$ (Abbott, Rungis, France) or the LIAISON ${ }^{\circledR}$ XL Murex HIV Ab/Ag HT (Diasorin, Antony, France). Furthermore, when primary HIV infection was suspected, concomitant plasma HIV-1 RNA was measured using a HIV1-RNA PCR with a RealTime ${ }^{\circledR}$ HIV-1assay (limit of quantification 40 copies/ml) (Abbott) or AmpliPrep/COBAS ${ }^{\circledR}$ TaqMan $^{\circledR}$ HIV-1 test v2.0 (limit of quantification 20 copies/ml) (Roche, Meylan, France). In case of a positive ELISA-4G result, HIV-1 RNA was retrospectively measured from frozen plasma stored at previous visit. The date of HIV diagnosis in the trial was the earliest date of the first positive test, HIV-1 RNA or EIA-4G.

Furthermore, frozen samples obtained at the date of HIV diagnosis were centralized at Saint Louis hospital and retested with the ARCHITECT ELISA-4G assay, the BioPlex 2200 HIV Ag-Ab assay (Biorad, Marnes-La-Coquette, France) with the p24 result separately from HIV antibodies and HIV-1 Western Blot $^{\circledast}$ (WB, Biorad, Marnes-La-Coquette, France). This allowed to define six stages, according to Fiebig 9.

\section{Study Oversight}

The conduct of the trial at each study site was monitored by the Service Commun 10-Unité de Service 19 (a Clinical Trial Unit) of INSERM. Gilead Sciences donated the study medications and provided funding for the pharmacokinetics analysis but had no role in data collection, data analysis, or manuscript preparation. All the authors vouch for the completeness and accuracy of the data reported and adherence to the study protocol.

\section{Study population}

All subjects enrolled in the modified-intent to treat population of the double-blind phase of the ANRS IPERGAY trial were eligible for this analysis. Follow-up was censored in case of HIV infection acquired during the blind phase, and for all other participants at their last visit of the blind phase. 
Because of the highly variable sexual activity and therefore pill intake within and between participants

160

161

162

163 over time, we grouped for the analysis the periods between visits during which participants had a similar behaviour, categorised in the three following patterns.

Periods during which participants took 15 pills or less per month but used them "systematically or often during sexual intercourses", a proxy for infrequent sexual intercourses covered by PrEP, were categorized in the pattern "high PrEP adherence with infrequent sexual intercourses". Two other behaviour patterns were defined: "low adherence to PrEP", defined by an uptake of 15 pills or less per month, taken from time to time or never during sexual intercourses; "high PrEP use", defined by an uptake of more than 15 pills per month. We then cumulated in each arm follow-up (FU) time spent with each behaviour pattern.

Our main analysis focused on periods of "high PrEP adherence with infrequent sexual intercourses".

\section{Estimation of timing of HIV infection}

For each HIV infection occurring during the trial, we blindly estimated the time of HIV acquisition without knowing the treatment arm and the pattern of PrEP use, using dates of positive plasma HIVRNA, ELISA-4G tests, results of Western blots and Fiebig staging. HIV-1 RNA becomes detectable in plasma on average 11 days following infection ${ }^{10,11}$; therefore, HIV infection was estimated to have occurred within 11 to 19.1 days prior to a Fiebig I diagnosis, 14.1 to 24.5 days prior to a Fiebig II, 18.1 to 28.0 days prior to a Fiebig III, 21.0 to 33.9 days for Fiebig IV, 26.3 to 140.8 days for Fiebig V, and more than 58.4 days before for a Fiebig VI diagnosis ${ }^{11}$ (Figure 1).

In some cases, the infection window period could even be shortened due to a tight sequence of a negative followed by a positive ELISA-4G or HIV-1 RNA: since HIV-1 RNA becomes detectable after around eleven days following infection and ELISA-4G becomes positive after around 16 days following infection $^{10,11}$, we considered that HIV infection could not have occurred more than 11 or 16 days respectively before the last negative HIV-1 RNA or ELISA-4G, nor less than 11 or 16 days respectively before the first positive HIV-1 RNA or ELISA-4G (figure 1). 
185

In the main analysis, HIV incidence rate was estimated as the ratio of the number of HIV-1 infections occurring during a period of high adherence to PrEP with infrequent sexual intercourses, to the total of person-years cumulated with this pattern of PrEP use. Incidence rates in the TDF/FTC and placebo arm were compared to assess the efficacy of the PrEP. The efficacy of the PrEP was also assessed for the two other patterns of PrEP use.

When the estimated time window of HIV infection spread over two different periods of pattern of PrEP use, the longest period was considered to be the one during which HIV infection occurred. A sensitivity analysis was performed, where infection was considered to have occurred during the alternative period.

Other analyses were performed, lowering the threshold from 15 pills to 10 pills per month in order to explore the efficacy of PrEP with an even more restrictive definition of infrequent sexual intercourses.

All analyses were conducted with R version 3.5.2. Exact confidence intervals and tests to compare HIV incidence rates between the two arms were obtained by the mid-p method, which avoids overconservative estimation of confidence intervals and $p$-values ${ }^{12}$. Relative risks (RR), relative reductions of risk (RRR), 95\% confidence intervals ( $\mathrm{Cl}$ ) and p-values were obtained using the package \{epitools\}, the function epitab(), and the following parameters: method="rateratio", rateratio="midp".

\section{Results}

Four hundred participants were included in the blind phase of the Ipergay trial, 201 in the placebo arm and 199 in the Truvada arm, for a total follow-up of 431 Person-Years (PY). The number of pills used per month was above 15 pills for 188 PY ("high PrEP use"), 15 pills or less for 180 PY, unknown for 63 PY. When the number of pills used per month was 15 pills or less, PrEP was reported to be used "systematically or often" during sexual intercourses for 134 PY ("high adherence to PrEP with infrequent sexual intercourses"), as opposed to "from time to time" or "not at all" ("low adherence", 
45 PY). Our main analysis focused on these 134 PY of high adherence to PrEP with infrequent sexual intercourses, representing $31 \%$ of the total follow-up of the Ipergay trial, and provided by 270 participants, 134 from the placebo arm and 136 from the TDF/FTC arm. Compared to the other 130 participants of the Ipergay trial who never experienced this pattern of PrEP use during follow-up, they had a higher level of education ( $77 \%$ of post-secondary education vs. $65 \%, p=0.02$ ) and reported at enrolment a lower number of sexual partners over the past 2 months (median 8 vs. 10, respectively, $p=0.002$ ) and of sexual intercourses over the past 4 weeks (median 10 vs. $12, p=0.03$ ). They did not differ for age or for bacterial sexually transmitted infection diagnosed at screening (supplementary table 1). The baseline characteristics of these 270 participants did not significantly differ between the two study arms, TDF/FTC versus Placebo (table 1).

During the periods of infrequent sexual intercourses with high adherence to PrEP, the median number of sexual intercourses per month was 5 [IQR: 2; 10] (range from 0 to $>100$ ), and the median number of pills used per month was 9.5 [IQR: 6; 13] (table 2). In the TDF/FTC arm, the percentage of plasma samples with unquantifiable TDF was $38 \%$; the median dosage when quantifiable was $41 \mu \mathrm{g} / \mathrm{L}$ [IQR: 11-92]. The corresponding figures during periods when the participants had a "low adherence" to PrEP were $8.5[4.0 ; 18.5]$ sexual intercourses/month, $0[0 ; 4]$ pills/month and $87 \%$ of unquantifiable TDF. In contrast, during periods when participants were using more than 15 pills per month ("high PrEP use"), they had a median of 12 [8-20] sexual intercourses per month and an intake of 23.5 [19-27] pills per month; $11 \%$ had unquantifiable TDF in plasma (median dosage [IQR] when quantifiable: $84 \mu \mathrm{g} / \mathrm{L}$ [39146]).

Among the 16 HIV-1 infections which occurred during the blind phase of the Ipergay trial (14 in the placebo arm and 2 in the TDF/FTC arm), 6 infections occurred during periods when pill use was 15 or less per month and PrEP was systematically or often taken during sexual intercourses (figure 2). These 6 participants had all been randomized in the placebo arm. The HIV-1 incidence rate was 9.2 per 100 
PY (95\% Cl: 3.4 - 20.1) in the placebo arm versus $0.0(0.0$ - 5.4) per $100 \mathrm{PY}$ in the TDF/FTC arm, $p=0.013$

233 (table 2); the relative reduction of incidence rate was $100 \%(95 \% \mathrm{Cl}: 39-100)$.

234

In one case (\#3), the infection window period slightly overlapped with the following period when he used more than 15 pills per month, actually $16 /$ month. A sensitivity analysis considering that this infection occurred when the number of pills used per month was more than 15 led to similar conclusions, with five infections with placebo and an incidence rate of 7.7 per $100 \mathrm{PY}$ (95\% Cl: 2.5 - 18) versus 0.0 with TDF/FTC arm, $\mathrm{p}=0.027$.

Other analyses assessing the efficacy of PrEP with lower thresholds of pill intake showed similar trends, although the difference was not always statistically significant, due to a lower number of events. For instance, when the analysis was restricted to periods when participants used 10 pills or less per month, taken systematically or often during sexual intercourses, HIV incidence was 7.86 per 100 PY [1.6; 23] in the placebo arm ( 3 infections during $38.2 \mathrm{PY}$ of $\mathrm{FU}$ ) and 0.0 per 100 py in the TDF/FTC arm ( 0 infection during 38.8 PY of $F U), p=0.12$.

No efficacy of TDF/FTC was observed when participants used 15 pills or less per month taken from time to time or not at all (table 2). The 2 cases of HIV-1 infections diagnosed in the TDF/FTC arm during the blind phase of the trial occurred in periods when the participants declared to have used PrEP not at all or from time to time: they had used 2.5 and 1 pills/month and had 6 and 12 sexual intercourses/month, respectively.

\section{Discussion}

This study showed that on-demand PrEP with TDF/FTC remains highly effective among MSM enrolled in the ANRS IPERGAY trial during periods when they reported systematic or frequent PrEP use and infrequent sexual intercourses, leading to an uptake of 15 pills or less per month. The chosen threshold of 15 pills per month led to consider periods of follow-up when the participants actually used a median of 9.5 pills per month and had a median of 5 sexual intercourses per month, i.e. around 2.2 pills and 
less than 1.2 intercourses per week, on average. During these periods of low pill intake due to

257 infrequent sexual exposure, the HIV-1 incidence rate with placebo was 9.2 per $100 \mathrm{PY}(95 \% \mathrm{Cl}: 3.4$ -

258 20.1), versus $0(0.0-5.4)$ with TDF/FTC, $p=0.013$.

Modelling studies from the iPrEx and STRAND studies indicated that TFD-dp concentration corresponding to a use of 4 pills/week gave a relative reduction of risk (RRR) of 96\% [95\% Cl: 90\%>99\%]. The RRR was 76\% [95\% Cl: 56\%-96\%] for 2 pills per week ${ }^{13}$. In addition, modelling from iPrEx OLE found that the TFV-dp concentration associated with $90 \%$ reduction risk was consistent with use of 2 to 3 tablets per week ${ }^{7}$. Here, we observed a RRR of $100 \%$ [95\% Cl: $39 \%-100 \%$ ] with an average uptake of 2.2 pills/week, provided that adherence to the on-demand PrEP regimen was good. Our results provide evidence of high efficacy of on-demand PrEP in case of infrequent periods of sexual intercourses, whatever the number of sexual acts during these periods.

The efficacy of the event-driven PrEP, even with an infrequent use, is consistent with both monkey studies and pharmacokinetic studies. Studies including groups of six rhesus macaques receiving different PrEP regimen showed that significant protection is achieved by event-driven PrEP of TDF/FTC 22 hours before and 2 hours before the exposure (4/6 uninfected macaques after 14 weekly virus challenge versus $0 / 6$ control receiving no PrEP) ${ }^{14}$. Of note, in another study with daily oral TDF/FTC, 4 out of 6 macaques were also still uninfected after 14 weekly challenges ${ }^{15}$ suggesting that in macaques, intermittent oral preexposure prophylaxis with TDF/FTC was as effective as daily prophylaxis.

Pharmacokinetic/pharmocodynamic studies in healthy women reinforce this conclusion. The proportion of the population that achieved the target of $90 \%$ effective concentration ratios of TFV-dp to dATP (EC90 TFVdp:dATP) in the colorectal tissue was $100 \%$ after 3 TDF/FTC daily doses and $>95 \%$ with a regimen of 2 doses/week ${ }^{16}$. Using an intermittent regimen based on the Ipergay protocol, $81 \%$ and $98 \%$ of the population achieved EC90 TFVdp:dATP at the time of the coitus when 2 TDF/FTC doses were administered 2 hours and 24 hours before coitus, respectively. Since no difference in colorectal 
drug concentration and risk of transmission during anal intercourses has been identified by sex, we can reasonably extrapolate these results to the MSM population.

This study is not strictly speaking a randomized comparison since the periods of infrequent PrEP uptake occurred after randomization; however, since the trial was double-blind, neither adherence to treatment nor sexual behaviour were dependent on the arm. We are therefore confident that the comparison between the arms is not biased.

We want to underline that the results are based on a large sample of the IPERGAY blind phase: twothirds of the Ipergay participants were at least once in the case of infrequent use due to infrequent sexual intercourses. Noteworthy, when 15 pills or less per month were taken, PrEP was systematically or often used during intercourses in $75 \%$ of the participation time. It appears therefore that an infrequent use of pills corresponded in the vast majority of participants to a reasoned choice based on the frequency of their sexual activity.

Among the strengths of this study is the precise estimation of the window period when HIV infection occurred. This was made possible through the high frequency of HIV testing during the Ipergay trial, at each study-visit, month 1 , month 2 and every 2 months thereafter, and the possibility to perform additional assays from frozen samples drawn at previous visits in order to date the infection more precisely.

Of note, the 2 cases of HIV-1 infections diagnosed in the TDF/FTC arm during the blind phase of the trial occurred in periods when the participants reported having used PrEP not at all or from time to time. Our results therefore confirm that among strongest determinants of on-demand PrEP efficacy is compliance to the regimen.

We acknowledge that due to a low number of person-years, the efficacy of on-demand PrEP with very low levels of PrEP uptake, such as once a month, could not be studied. Future open studies of ondemand PrEP will help to clarify this issue. 
In conclusion, these data provide evidence that sex-driven PrEP is an adequate alternative to daily PrEP for high-risk MSM even during periods of less frequent sexual intercourses. The choice of the regimen (daily or sex-driven) should be offered to all MSM subjects, who could therefore adapt their uptake according to their evolving sexual lives and more broadly to their lives context.

\section{Contributors}

L.M, J-M.M, G.A and C.T designed the study. G.A wrote the first draft of the report. GA, LM and IC designed the analysis. G.A, C.D, L.M, I.C, C.C, and J-M.M analysed the data. C.C coordinated data management. C.D, E.C, F.R, T.H, L.C, J.C, C.T and J-M.M did the study at their sites. B.S and D.R organized the community support of the participants. All authors critically reviewed and approved the manuscript.

\section{Declaration of interests}

J-MM reports receiving support as an adviser for Gilead Sciences, Merck, ViiV Healthcare and Teva, and research grants from Gilead Sciences. CD reports receiving support as an adviser for Gilead, Merck, and Janssen, and research grants from Gilead and ViiV Healthcare.

\section{Acknowledgments}

We are grateful to the study participants for their trust in the study, the National Agency for Research on AIDS and Hepatitis (ANRS; France Recherche Nord \& Sud Sida-HIV Hépatites) and its Director François Dabis, Canadian HIV Trials Network, Fondation Pierre Bergé pour la prevention/Sidaction, and the Bill \& Melinda Gates Foundation for their grant support, to Gilead Sciences for donation of tenofovir disoproxil fumarate and emtricitabine, and to Jean-François Delfraissy for his support from the beginning of the trial. We thank the gay communities in France and Canada (AIDES, COQSIDA, REZO) who supported this work.

The ANRS IPERGAY Study Team includes the authors of this report and the following: 
INSERM SC10-US19: L Meyer, C Capitant, I Charreau, E Netzer, N Leturque, J Binesse, V Foubert, M

328 Saouzanet, F Euphrasie, D Carette, B Guillon, Y Saïdi, and J P Aboulker.

329 INSERM UMR 912 SESSTIM: B Spire, M Suzan, G Cattin, B Demoulin, L Sagaon-Teyssier, and N Lorente.

330 ANRS: V Doré, E Choucair, S Le Mestre, A Mennecier, N Etien, M C Simon, A Diallo, S Gibowski, and J F

331 Delfraissy.

332 Rezo Canada: D Thompson.

333 The Canadian HIV Trials Network: J Sas, J Pankovitch, M Klein, and A Anis.

334 Members of the Scientific Committee: Jean-Michel Molina (Chair), Mark A Wainberg, Benoit Trottier, 335 Cécile Tremblay, Jean-Guy Baril, Gilles Pialoux, Laurent Cotte, Antoine Chéret, Armelle Pasquet, Eric 336 Cua, Michel Besnier, Willy Rozenbaum, Christian Chidiac, Constance Delaugerre, Nathalie Bajos, Julie 337 Timsit, Gilles Peytavin, Julien Fonsart, Isabelle Durand-Zaleski, Laurence Meyer, Jean-Pierre Aboulker, 338 Bruno Spire, Marie Suzan-Monti, Gabriel Girard, Daniela Rojas Castro, Marie Préau, Michel Morin, 339 David Thompson, Catherine Capitant, Anaïs Mennecier, Elias Choucair, Véronique Doré, Marie340 Christine Simon, Isabelle Charreau, Joanne Otis, France Lert, Alpha Diallo, Séverine Gibowski, and 341 Cecile Rabian. 
1. Grant, R. M. et al. Preexposure chemoprophylaxis for HIV prevention in men who have sex with men. N. Engl. J. Med. 363, 2587-2599 (2010).

2. McCormack, S. et al. Pre-exposure prophylaxis to prevent the acquisition of HIV-1 infection (PROUD): effectiveness results from the pilot phase of a pragmatic open-label randomised trial. Lancet 387, 53-60 (2016).

3. Molina, J.-M. et al. On-Demand Preexposure Prophylaxis in Men at High Risk for HIV-1 Infection. N. Engl. J. Med. 373, 2237-2246 (2015).

4. Europen AIDS Clinical Society, Guidelines, http://www.eacsociety.org/files/guidelines_9.0english.pdf.

5. https://www.cdc.gov/hiv/pdf/risk/prep/cdc-hiv-prep-guidelines-2017.pdf.

6. WHO | Guidance on oral pre-exposure prophylaxis (PrEP) for serodiscordant couples, men and transgender women who have sex with men at high risk of HIV. WHO Available at: https://www.who.int/hiv/pub/guidance_prep/en/. (Accessed: 11th February 2019)

7. Grant, R. M. et al. Uptake of pre-exposure prophylaxis, sexual practices, and HIV incidence in men and transgender women who have sex with men: a cohort study. Lancet Infect Dis 14, 820-829 (2014).

8. Fonsart, J. et al. Single-dose pharmacokinetics and pharmacodynamics of oral tenofovir and emtricitabine in blood, saliva and rectal tissue: a sub-study of the ANRS IPERGAY trial. J. Antimicrob. Chemother. 72, 478-485 (2017).

9. Delaugerre, C. et al. Assessment of HIV Screening Tests for Use in Preexposure Prophylaxis Programs. J. Infect. Dis. 216, 382-386 (2017).

10. Delaney, K. P. et al. Time Until Emergence of HIV Test Reactivity Following Infection With HIV-1: Implications for Interpreting Test Results and Retesting After Exposure. Clin. Infect. Dis. 64, 53-59 (2017).

11. Fiebig, E. W. et al. Dynamics of HIV viremia and antibody seroconversion in plasma donors: implications for diagnosis and staging of primary HIV infection. AIDS 17, 1871-1879 (2003).

12. Berry, G \& Armitage, P. Mid-P confidence. The Statistician 44, 417-423 (1995). 
13. Anderson, P. L. et al. Emtricitabine-tenofovir concentrations and pre-exposure prophylaxis efficacy in men who have sex with men. Sci Transl Med 4, 151ra125 (2012).

14. García-Lerma, J. G. et al. Intermittent prophylaxis with oral truvada protects macaques from rectal SHIV infection. Sci Transl Med 2, 14ra4 (2010).

15. García-Lerma, J. G. et al. Prevention of rectal SHIV transmission in macaques by daily or intermittent prophylaxis with emtricitabine and tenofovir. PLoS Med. 5, e28 (2008).

16. Cottrell, M. L. et al. A Translational Pharmacology Approach to Predicting Outcomes of Preexposure Prophylaxis Against HIV in Men and Women Using Tenofovir Disoproxil Fumarate With or Without Emtricitabine. J. Infect. Dis. 214, 55-64 (2016). 
Table 1: Baseline characteristics of the $\mathbf{2 7 0}$ participants who had at least once during follow-up periods of infrequent sexual intercourses with high adherence to on-demand PrEP regimen: : the ANRS Ipergay trial

\begin{tabular}{|c|c|c|c|}
\hline Baseline characteristics & Placebo ( $\mathrm{N}=134)$ & TDF/FTC (N=136) & $P$ value \\
\hline Median age [IQR] & $34[28-42]$ & $35[29-43]$ & 0.88 \\
\hline White Race & & & 0.08 \\
\hline No -no.(\%) & $15(11 \%)$ & $7(5 \%)$ & \\
\hline Yes -no.(\%) & $119(89 \%)$ & $129(95 \%)$ & \\
\hline Relationship status & & & 0.80 \\
\hline Does not live with his partner -no.(\%) & $100(81 \%)$ & $98(78 \%)$ & \\
\hline Lives with a HIV positive partner -no.(\%) & $8(7 \%)$ & $11(9 \%)$ & \\
\hline Lives with a HIV negative partner -no.(\%) & $15(12 \%)$ & $16(13 \%)$ & \\
\hline Missing -no. & 11 & 11 & \\
\hline Post-secondary education & & & 0.47 \\
\hline No -no.(\%) & $34(25 \%)$ & $29(21 \%)$ & \\
\hline Yes -no.(\%) & $100(75 \%)$ & $106(79 \%)$ & \\
\hline Missing -no. & 0 & 1 & \\
\hline Use of recreational drugs(2) & & & 0.62 \\
\hline No -no.(\%) & $69(53 \%)$ & $76(56 \%)$ & \\
\hline Yes -no.(\%) & $62(47 \%)$ & $59(44 \%)$ & \\
\hline Missing -no. & 3 & 1 & \\
\hline At least 5 glasses a typical day of drinking & & & 0.15 \\
\hline No -no.(\%) & $109(81 \%)$ & $100(74 \%)$ & \\
\hline Yes -no.(\%) & $25(19 \%)$ & $36(26 \%)$ & \\
\hline Site of enrolment & & & 0.82 \\
\hline Paris -no.(\%) & $72(54 \%)$ & $70(51 \%)$ & \\
\hline Lyon -no.(\%) & $28(21 \%)$ & $34(25 \%)$ & \\
\hline Nice -no.(\%) & $11(8 \%)$ & $7(5 \%)$ & \\
\hline Tourcoing -no.(\%) & $9(7 \%)$ & $7(5 \%)$ & \\
\hline Nantes -no.(\%) & $5(4 \%)$ & $6(4 \%)$ & \\
\hline Montreal -no.(\%) & $9(7 \%)$ & $12(9 \%)$ & \\
\hline $\begin{array}{l}\text { Median no. of sexual partners in past } 2 \text { months } \\
\text { [IQR] }\end{array}$ & $8[4-15]$ & $8[4-15]$ & 0.84 \\
\hline $\begin{array}{l}\text { Median no. of episodes of sexual intercourses in } \\
\text { past } 4 \text { weeks [IQR] (Missing) }\end{array}$ & $10[4-15](1)$ & $10[5-15](2)$ & 0.62 \\
\hline Circumcision & & & 0.65 \\
\hline No -no.(\%) & $105(78 \%)$ & $110(81 \%)$ & \\
\hline Yes -no.(\%) & $29(22 \%)$ & $26(19 \%)$ & \\
\hline $\begin{array}{l}\text { Bacterial sexually transmitted infection diagnosed } \\
\text { at screening(3) }\end{array}$ & & & 0.22 \\
\hline No -no.(\%) & $93(69 \%)$ & $104(76 \%)$ & \\
\hline Yes -no.(\%) & $41(31 \%)$ & $32(24 \%)$ & \\
\hline
\end{tabular}

(1) Non parametric Wilcoxon and Fisher tests for continuous and qualitative characteristics, respectively.

(2) Recreational drugs in past 12 months including ecstasy, crack, cocaine, crystal, speed, GHB/GBL

(3) Infections included Syphilis (as detected on serologic testing by means of rapid plasma reagin confirmed with the use of a treponema-specific assay), N. gonorrhoeae and C. trachomatis (as detected on PCR on urine samples, throat or anal swabs)

Periods of infrequent sexual intercourses with high adherence to PrEP were defined as a PrEP intake of 15 pills or less per month with a systematic or frequent use during sexual intercourses. 
Table 2: Efficacy of on-demand PrEP according to the pattern of use: : the ANRS Ipergay trial

\begin{tabular}{|c|c|c|c|c|c|c|c|}
\hline & $\begin{array}{l}\text { Number of sexual } \\
\text { intercourses/month } \\
\text { (median [IQR]) }\end{array}$ & $\begin{array}{l}\text { Number of } \\
\text { pills/month } \\
\text { (median [IQR]) }\end{array}$ & $\begin{array}{c}\text { TFV } \\
\text { concentration } \\
\text { (ng/mL, } \\
\text { median [IQR]) }\end{array}$ & $\begin{array}{l}\text { Person- } \\
\text { Years }\end{array}$ & $\begin{array}{c}\text { Number } \\
\text { of HIV1 } \\
\text { infections }\end{array}$ & $\begin{array}{l}\text { HIV1 Incidence } \\
\text { Rate }\end{array}$ & $\mathbf{p}$ \\
\hline High PrEP use (2) & $12[8-20]$ & 23.5 [19-27] & & 187.8 & 7 & & \\
\hline Placebo & & & & 86.4 & 7 & $8.1[3.3 ; 16.7]$ & \\
\hline TDF/FTC & & & $72[20-133]$ & 101.4 & 0 & $0.0[0 ; 3.6]$ & 0.0044 \\
\hline Low PrEP adherence (3) & $8.5[4-18.5]$ & $0[0-4]$ & & 45.3 & 2 & & \\
\hline Placebo & & & & 25.6 & 0 & $0.0[0 ; 14.4]$ & \\
\hline TDF/FTC & & & $0[0-18]$ & 29.1 & 0 & $0.0[0 ; 12.7]$ & 0.55 \\
\hline
\end{tabular}

(1) $\leq 15$ pills/month taken systematically or often during sexual intercourses

(2) $>15$ pills/month

(3) $\leq 15$ pills/month taken from time to time or never during sexual intercourses

(4) Bottles not returned or question about compliance not answered with 15 pills or less/month.

(5) One infected participant from the placebo arm could not be categorized because he did not return the bottles. He reported 20 sexual acts in the previous period and a systematic use of PrEP during sexual intercourses. 
Supplementary table 1: Comparison of baseline characteristics of infrequent PrEP users with other Ipergay participants: : the ANRS Ipergay trial

\begin{tabular}{|c|c|c|c|}
\hline Baseline characteristics & $\begin{array}{c}\text { infrequent } \\
\text { PrEP } \\
\text { users } \\
\text { (1) } \\
\mathbf{N}=\mathbf{2 7 0}\end{array}$ & $\begin{array}{c}\text { other } \\
\text { participants } \\
\mathrm{N}=130\end{array}$ & p value ${ }^{(2)}$ \\
\hline Median age [IQR] - years & $34[28-42]$ & 36 [30-43] & 0.34 \\
\hline White - no. (\%) & 248 (92\%) & $118(91 \%)$ & 0.71 \\
\hline Post-secondary education - no. (\%) & 206 (77\%) & $81(65 \%)$ & 0.02 \\
\hline Relationship status - no (\%) & & & 0.51 \\
\hline Not in a couple & $198(73 \%)$ & 95 (73\%) & \\
\hline In a couple with HIV-1-positive partner & $19(7 \%)$ & $13(10 \%)$ & \\
\hline Other & $53(20 \%)$ & $22(17 \%)$ & \\
\hline$>5$ alcoholic drinks per day at least once in past month - no. (\%) & $61(24 \%)$ & $30(26 \%)$ & 0.70 \\
\hline Use of recreational drugs ${ }^{(3)}-$ no. (\%) & $121(45 \%)$ & $56(47 \%)$ & 0.91 \\
\hline Site of enrolment- no. (\%) & & & 0.04 \\
\hline Lyon & $62(23 \%)$ & $21(16 \%)$ & \\
\hline Montreal & $21(8 \%)$ & $22(17 \%)$ & \\
\hline Nantes & $11(4 \%)$ & $4(3 \%)$ & \\
\hline Nice & $18(7 \%)$ & $13(10 \%)$ & \\
\hline Paris & $142(53 \%)$ & $59(45 \%)$ & \\
\hline Tourcoing & $16(6 \%)$ & $11(8 \%)$ & \\
\hline Median no. of sexual partners in past 2 months [IQR] & $8[4-15]$ & $10[6-20]$ & 0.002 \\
\hline $\begin{array}{l}\text { Median no. of episodes of sexual intercourses in past } 4 \text { weeks } \\
\text { [IQR] }\end{array}$ & $10[5-15]$ & $12[6-20]$ & 0.03 \\
\hline Circumcision- no. (\%) & $55(20 \%)$ & 24 (18\%) & 0.69 \\
\hline $\begin{array}{l}\text { Bacterial sexually transmitted infection diagnosed at screening }{ }^{(4)}- \\
\text { no. (\%) }\end{array}$ & $73(27 \%)$ & $38(29 \%)$ & 0.72 \\
\hline
\end{tabular}

(1) Defined by at least once an uptake of 15 pills or less per month with a systematic or often use during intercourses

(2) Non parametric tests: Wilcoxon and Fisher test for continuous and qualitative characteristics, respectively.

(3) Recreational drugs in past 12 months including ecstasy, crack, cocaine, crystal, speed, GHB/GBL

(4) Infections included Syphilis (as detected on serologic testing by means of rapid plasma reagin confirmed with the use of a treponema-specific assay), N. gonorrhoeae and C. trachomatis (as detected on PCR on urine samples, throat and anal swabs) 
Figure 1. Estimation of the time interval during which the infection occurred using dates of HIVRNA conversion, of ELISA-4G conversion, and results of Western blot and Fiebig staging
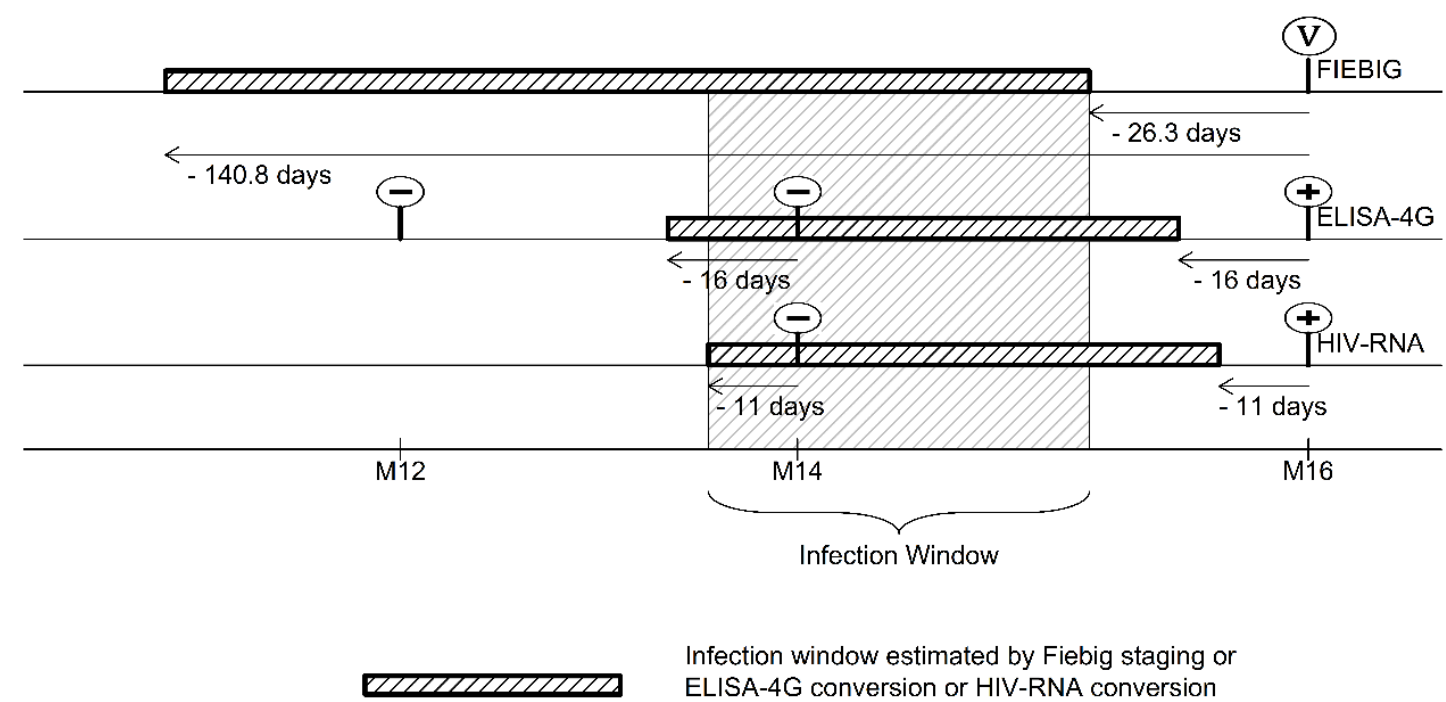

Infection window estimated by Fiebig staging or ELISA-4G conversion or HIV-RNA conversion

Infection window compatible with both Fiebig staging and ELISA-4G conversion and HIV-RNA conversion

HIV infection was estimated to have occurred within 11 to 19.1 days prior to a Fiebig I diagnosis, 14.1 to 24.5 days prior to a Fiebig II, 18.1 to 28.0 days prior to a Fiebig III, 21.0 to 33.9 days for Fiebig IV, 26.3 to 140.8 days for Fiebig V, and more than 58.4 days before for a Fiebig VI diagnosis ${ }^{10}$. Furthermore, HIV infection could not have occurred more than 11 or 16 days respectively before the last negative HIV-1 RNA or ELISA-4G, nor less than 11 or 16 days respectively before the first positive HIV-1 RNA or ELISA-4G ${ }^{9}$. In this example, the diagnosis was made in M16 at the Fiebig V stage with an infection period ranging from -140.8 days to -26.3 days before M16. This period of contamination could be shortened by the knowledge of an undetectable viral load at M14. Thus, the period of infection compatible with both Fiebig staging and ELISA-4G conversion and HIV-RNA conversion ranges from 11 days before M14 to -26.3 days before M16. 
Figure 2. Infection windows for the six participants infected during a period between two visits when 15 pills or less per month systematically or often during sexual intercourse were used (all in Placebo arm)
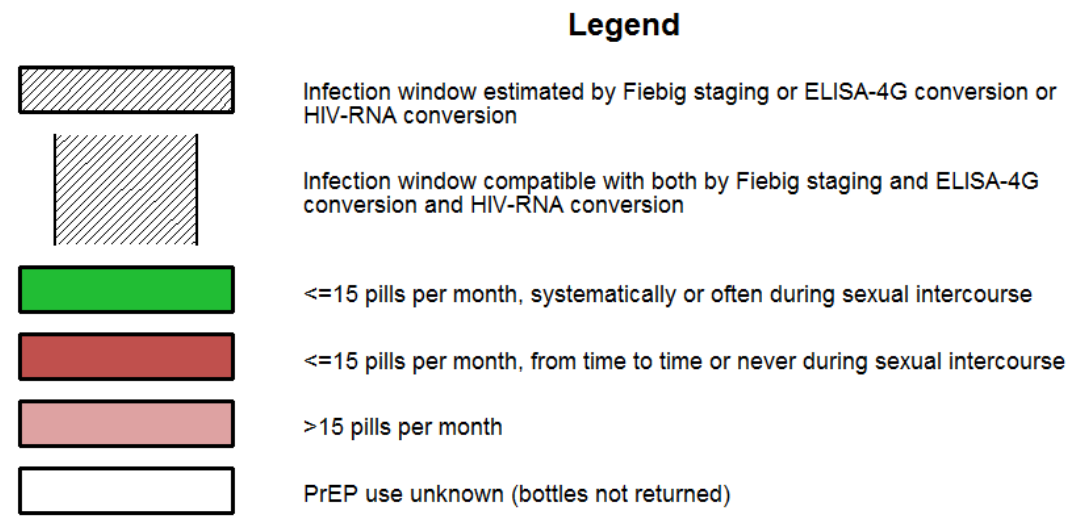

\#1

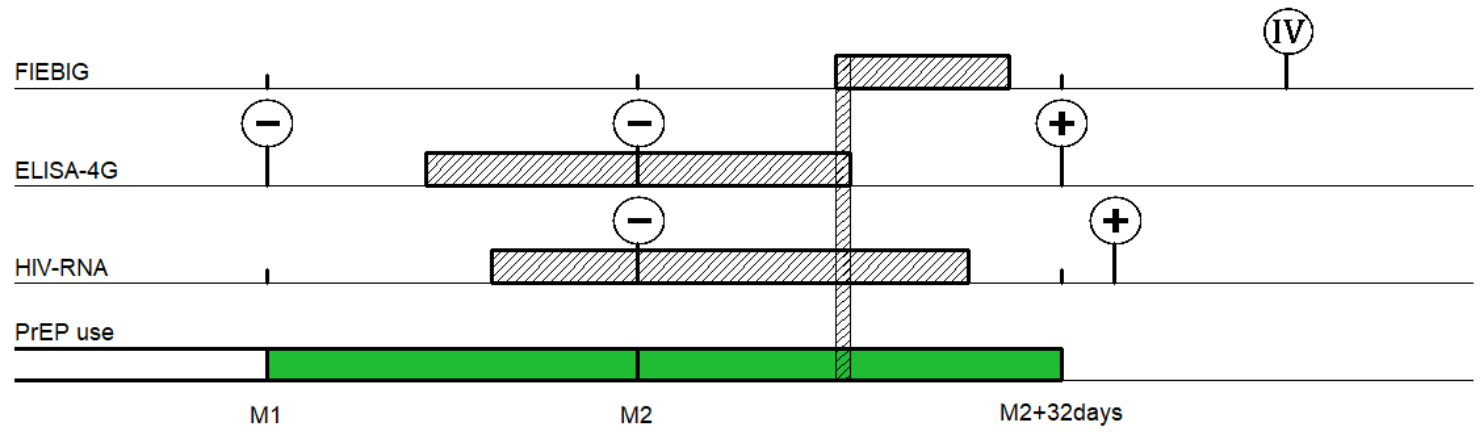

\#2

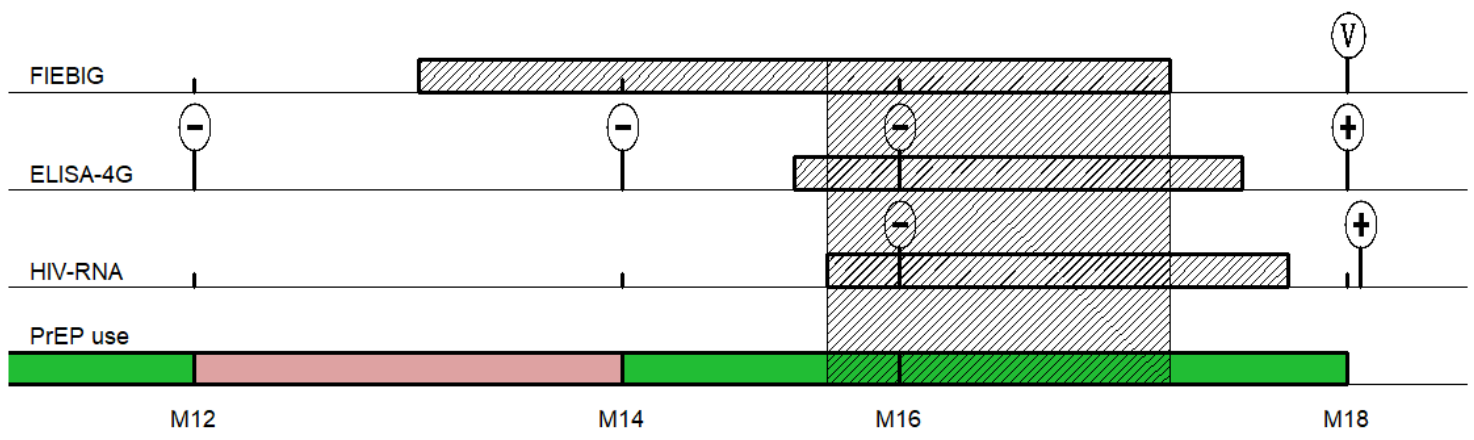

\#3

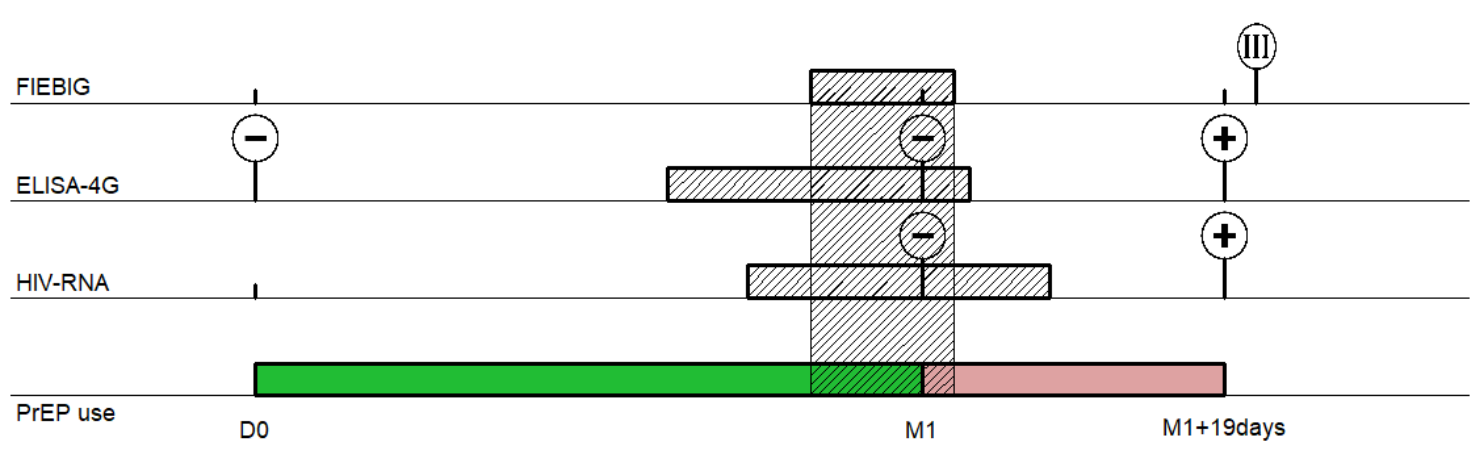




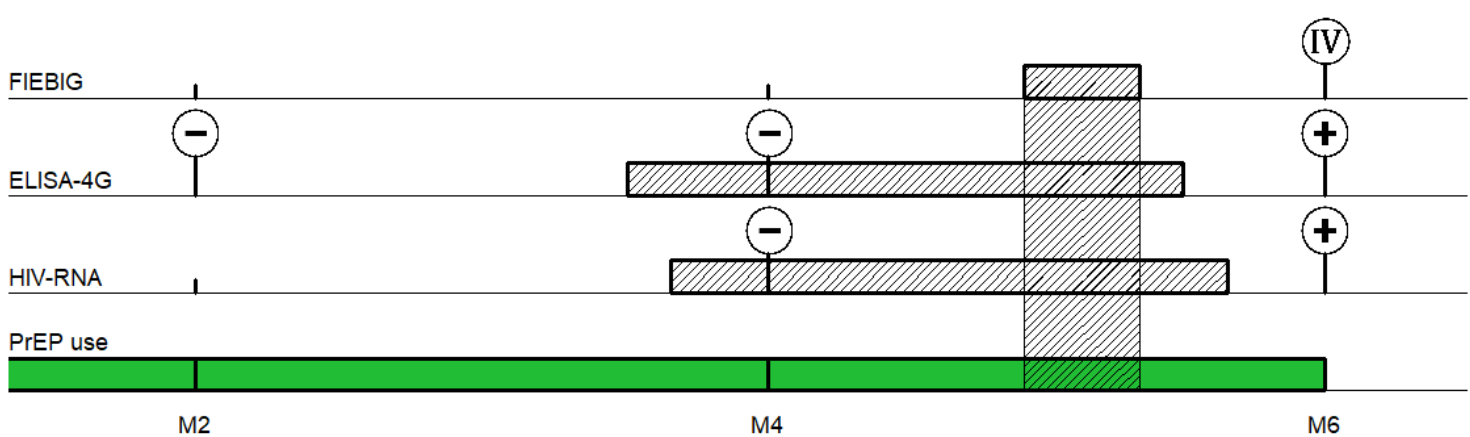

\#5

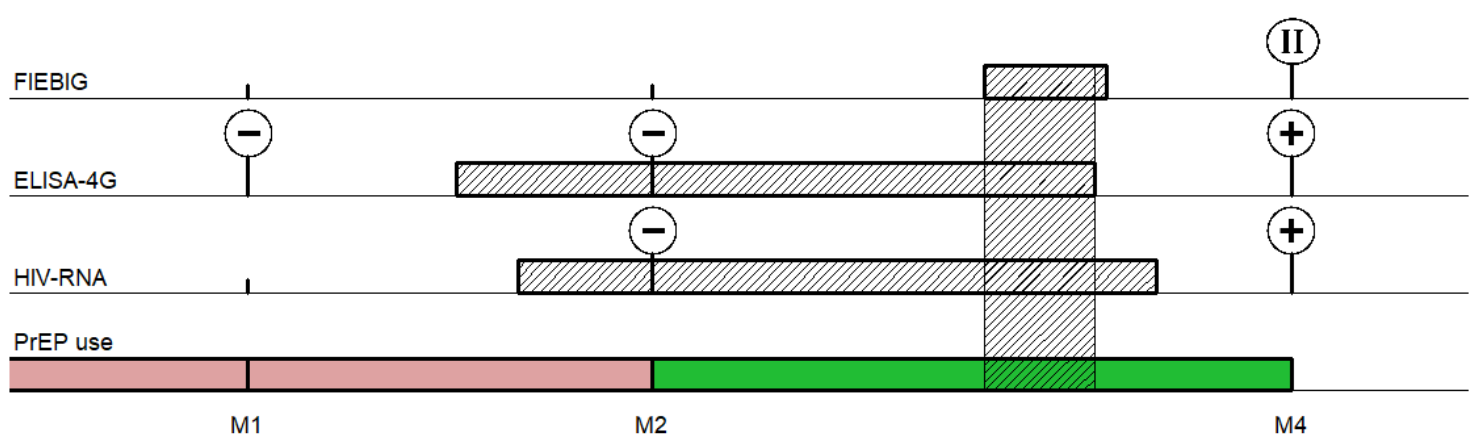

\#6

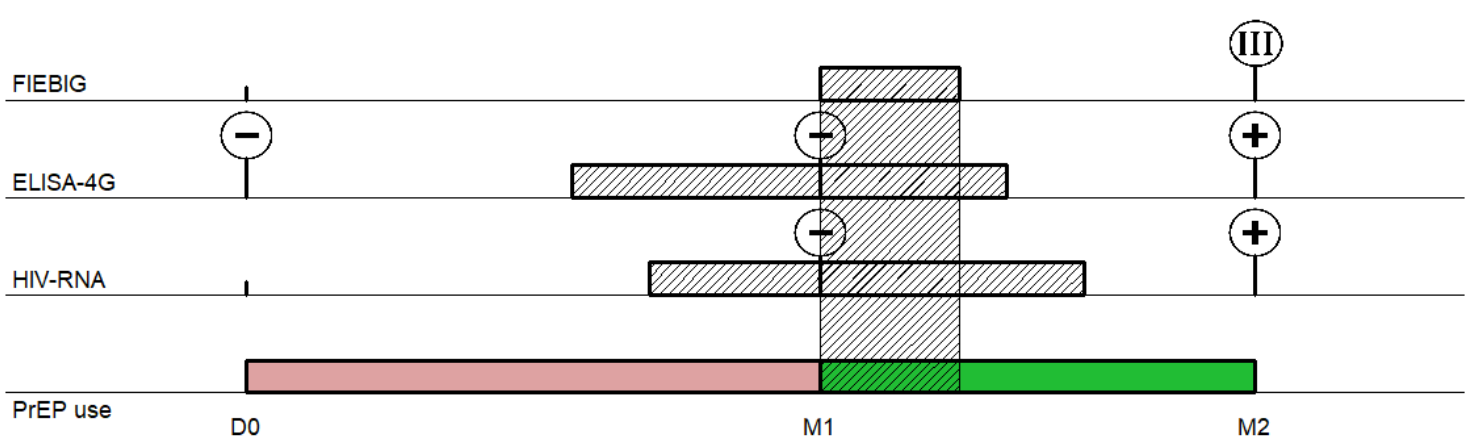

\#1 : HIV-RNA 5529 cp, EIA-4G Agp24- Ab+, undetermined Western-Blot (Fiebig IV); \#2 : HIV-RNA 9198 cp, EIA-4G Agp24- Ab+, Positive Western-Blot without p34 Ab (Fiebig V); \#3 : HIV-RNA 9346500 cp, EIA-4G Agp24+ Ab+, Negative Western-Blot (Fiebig III); \#4 : HIV-RNA 20672 cp, EIA-4G Agp24- Ab+, undetermined Western-Blot (Fiebig IV); \#5 : HIV-RNA 4774294 cp, EIA-4G Agp24+ Ab-, Negative Western-Blot (Fiebig II) ; \#6 : HIV-RNA 8985536 cp, EIA-4G Agp24+ Ab+, Negative Western-Blot (Fiebig III). 